\title{
Low order nonconforming finite element method for time-dependent nonlinear Schrödinger equation
}

\author{
Chao Xu' ${ }^{1}$, Jiaquan Zhou', Dongyang Shi ${ }^{2^{*}}$ (D) and Houchao Zhang ${ }^{3}$
}

\section{"Correspondence:}

zzshidy@126.com

${ }^{2}$ School of Mathematics and

Statistics, Zhengzhou University,

Zhengzhou, China

Full list of author information is

available at the end of the article

\section{Springer}

\begin{abstract}
The main aim of this paper is to apply a low order nonconforming $E Q_{1}^{\text {rot }}$ finite element to solve the nonlinear Schrödinger equation. Firstly, the superclose property in the broken $H^{1}$-norm for a backward Euler fully-discrete scheme is studied, and the global superconvergence results are deduced with the help of the special characters of this element and the interpolation postprocessing technique. Secondly, in order to reduce computing cost, a two-grid method is developed and the corresponding superconvergence error estimates are obtained. Finally, a numerical experiment is carried out to confirm the theoretical analysis.
\end{abstract}

MSC: $65 \mathrm{~N} 15 ; 65 \mathrm{~N} 30$

Keywords: Nonlinear Schrödinger equation; Two-grid method; Low order nonconforming finite element; Superconvergence error estimates

\section{Introduction}

Consider the following nonlinear Schrödinger equation (NLSE):

$$
\begin{cases}i u_{t}+\Delta u+\lambda f\left(|u|^{2}\right) u=0, & (X, t) \in \Omega \times(0, T], \\ u(X, t)=0, & (X, t) \in \partial \Omega \times(0, T], \\ u(X, 0)=u_{0}(X), & X \in \Omega,\end{cases}
$$

where $X=(x, y), \Omega \subset R^{2}$ is a bounded convex domain with Lipschitz boundary $\partial \Omega . i$ is the imaginary unit, $u(X, t)$ is a complex-valued function, $T \in(0,+\infty)$ is a real parameter, $f\left(|u|^{2}\right)=|u|^{2 r}$ ( $r \geq 1$ is an integer) is a smooth real-valued function, and $u_{0}(X)$ is a known smooth function.

The Schrödinger equation may describe many physical phenomena in optics, mechanics, and plasma physics, and it plays a very important role in various areas of mathematical physics. Numerical methods for this problem have been investigated extensively, e.g., see [1-3] for finite difference methods, [4-8] for finite element methods (FEMs), and [9-11] for others. Especially, the superconvergence analysis of FEMs for the Schrödinger equation have been studied successfully. For example, [12] used the conforming bilinear element to solve the LSE and obtained the superclose and superconvergence results in $H^{1}$-norm for

(c) The Author(s) 2018. This article is distributed under the terms of the Creative Commons Attribution 4.0 International License (http://creativecommons.org/licenses/by/4.0/), which permits unrestricted use, distribution, and reproduction in any medium, provided you give appropriate credit to the original author(s) and the source, provide a link to the Creative Commons license, and indicate if changes were made. 
the semi-discrete scheme. [13] derived the same results as [12] for NLSE with the conforming linear triangular element by establishing the relationship between Ritz projection and the linear interpolation. Whereafter, a series of superconvergence results about backward Euler and Crank-Nicolson fully-discrete schemes for NLSE also were studied in [14-17]. A two-grid method was first introduced by $\mathrm{Xu}[18,19]$ as a discretization technique for nonlinear and nonsymmetric indefinite partial differential equations. The main idea of this method is to use a coarse space (with mesh size $H$ ) to produce a rough approximation of the solution, and then use it as the initial guess for one Newton iteration on the fine grid (with mesh size $h$ and $h \ll H$ ). Up to now, the two-grid method was deeply researched for different problems [20-25]. Especially, the two-grid method was used to solve the linear Schrödinger equation (LSE) and NLSE in [26-30]. However, this method is rarely considered for nonconforming elements.

As we know, $E Q_{1}^{\text {rot }}$ element is an important quadrilateral nonconforming finite element and has been employed to deal with different problems successfully for its good theoretical and numerical behavior [31-37]. The purpose of this work is to use this element to deal with problem (1.1). By virtue of the special properties of this element, we obtain the superclose and superconvergence results in the broken $H^{1}$-norm for the backward Euler fully-discrete scheme. At the same time, in order to reduce the computing cost, we develop a new two-grid algorithm and deduce the corresponding superconvergence results.

The paper is organized as follows. In Sect. 2, $E Q_{1}^{\text {rot }}$ element and some lemmas are briefly introduced. In Sect. 3, the backward Euler fully-discrete scheme for problem (1.1) is discussed and some important superconvergence results are derived. In Sect. 4, a two-grid scheme of (1.1) is established and the corresponding superconvergence results are obtained. Finally, a numerical experiment is carried out to confirm the theoretical results.

\section{Nonconforming $E Q_{1}^{\text {rot }}$ element and some lemmas}

For simplicity, let $\Omega \subset \mathbb{R}^{2}$ be a convex polygon with edges parallel to the coordinate axes, $T_{h}$ be a regular subdivision of $\Omega$. For a given element $K$ with the center point $\left(x_{K}, y_{K}\right)$, its four vertices and edges are denoted as $a\left(x_{i}, y_{i}\right)(i=1,2,3,4)$ and $F_{i}=\overline{a_{i} a_{i+1}}(i=1,2,3$, $4 \bmod 4)$, respectively. We assume that edges $F_{i}(i=1,3)$ parallel to $x$-axis and $F_{i}(i=2,4)$ parallel to $y$-axis, $h_{x, K}$ and $h_{y, K}$ denote the half length of element $K$ along $x$ and $y$-axis, respectively.

The $E Q_{1}^{\text {rot }}$ finite element $(K, P, \Sigma)$ on $K$ is defined as follows:

$$
\Sigma=\left\{v_{1}, v_{2}, v_{3}, v_{4}, v_{5}\right\}, \quad P=\operatorname{span}\{1, x, y, \varphi(x), \varphi(y)\},
$$

where

$$
v_{i}=\frac{1}{\left|F_{i}\right|} \int_{F_{i}} v d s \quad(i=1,2,3,4), \quad v_{5}=\frac{1}{|K|} \int_{K} v d x d y, \varphi(t)=\frac{1}{2}\left(3 t^{2}-1\right),
$$

and $\left|F_{i}\right|$ and $|K|$ are the measures of $F_{i}$ and $K$, respectively.

The associated finite element space $V_{h}$ can be defined by

$$
V_{h}=\left\{v_{h}:\left.v_{h}\right|_{K} \in P, \forall K \in T_{h}, \int_{F}\left[v_{h}\right] d s=0, F \subset \partial K\right\},
$$

where $\left[v_{h}\right]$ denotes the jump value of $v_{h}$ across the boundary $F$, and $\left[v_{h}\right]=v_{h}$ if $F \subset \partial \Omega$. 
Obviously, $\|\cdot\|_{h}=\left(\sum_{K \in T_{h}}|\cdot|_{1, K}^{2}\right)^{\frac{1}{2}}$ is a norm over $V_{h}$.

Let $\Pi_{h}$ be the associated interpolation operator over $V_{h}$, then we have

$$
\left\|u-\Pi_{h} u\right\|_{0}+h\left\|u-\Pi_{h} u\right\|_{h} \leq C h^{2}|u|_{2}, \quad \forall u \in H^{2}(\Omega) \cap H_{0}^{1}(\Omega) .
$$

Lemma $2.1([31,32])$ For $v_{h} \in V_{h}$, we have

$$
\left|\sum_{K \in T_{h}} \int_{\partial K} \frac{\partial u}{\partial n} v_{h} d s\right| \leq \begin{cases}C h|u|_{2}\left\|v_{h}\right\|_{h}, & \forall u \in H^{2}(\Omega), \\ C h^{2}|u|_{3}\left\|v_{h}\right\|_{h}, & \forall u \in H^{3}(\Omega) .\end{cases}
$$

Lemma 2.2 For $v_{h} \in V_{h}$, we have

$$
\left|\sum_{K \in T_{h}} \int_{\partial K} \frac{\partial u}{\partial n} v_{h} d s\right| \leq C h^{2}|u|_{4}\left\|v_{h}\right\|_{0}, \quad \forall u \in H^{4}(\Omega) .
$$

Proof By introducing two functions

$$
E(x)=\frac{1}{2}\left(\left(x-x_{K}\right)^{2}-h_{x, K}^{2}\right), \quad F(y)=\frac{1}{2}\left(\left(y-y_{K}\right)^{2}-h_{y, K}^{2}\right),
$$

and notation $\left.P_{0} v_{h}\right|_{F_{i}}=\frac{1}{\left|F_{i}\right|} \int_{F_{i}} v_{h} d s$, which has continuity between elements and vanishes on $\partial \Omega$, and hence the summation

$$
\sum_{K \in T_{h}}\left(\int_{F_{2}}-\int_{F_{4}}\right) u_{x} P_{0} v_{h} d y=0, \quad \sum_{K \in T_{h}}\left(\int_{F_{3}}-\int_{F_{1}}\right) u_{y} P_{0} v_{h} d x=0 .
$$

So we can obtain that

$$
\begin{aligned}
& \sum_{K \in T_{h}} \int_{\partial K} \frac{\partial u}{\partial n} v_{h} d s \\
& =\sum_{K \in T_{h}}\left(\int_{F_{2}}-\int_{F_{4}}\right) u_{x} v_{h} d y+\sum_{K \in T_{h}}\left(\int_{F_{3}}-\int_{F_{1}}\right) u_{y} v_{h} d x \\
& =\sum_{K \in T_{h}}\left(\int_{F_{2}}-\int_{F_{4}}\right) u_{x}\left(v_{h}-P_{0} v_{h}\right) d y+\sum_{K \in T_{h}}\left(\int_{F_{3}}-\int_{F_{1}}\right) u_{y}\left(v_{h}-P_{0} v_{h}\right) d x \\
& =\sum_{K \in T_{h}} \int_{K}\left[u_{x x}\left(\left(y-y_{K}\right) v_{h y}+\left(\left(y-y_{K}\right)^{2}-\frac{h_{y, K}^{2}}{3}\right) \frac{v_{h y y}}{2}\right)\right] d x d y \\
& \quad+\sum_{K \in T_{h}} \int_{K}\left[u_{y y}\left(\left(x-x_{K}\right) v_{h x}+\left(\left(x-x_{K}\right)^{2}-\frac{h_{x, K}^{2}}{3}\right) \frac{v_{h x x}}{2}\right)\right] d x d y \\
& \triangleq A_{1}+A_{2},
\end{aligned}
$$

where we use the expressions

$$
\begin{aligned}
& \left.\left(v_{h}-P_{0} v_{h}\right)\right|_{F_{i}}=\left(y-y_{K}\right) v_{h y}+\left(\left(y-y_{K}\right)^{2}-\frac{h_{y, K}^{2}}{3}\right) \frac{v_{h y y}}{2}, \quad i=2,4, \\
& \left.\left(v_{h}-P_{0} v_{h}\right)\right|_{F_{i}}=\left(x-x_{K}\right) v_{h x}+\left(\left(x-x_{K}\right)^{2}-\frac{h_{x, K}^{2}}{3}\right) \frac{v_{h x x}}{2}, \quad i=1,3 .
\end{aligned}
$$


Now we begin to estimate $A_{1}$, which can be rewritten as

$$
\begin{aligned}
A_{1}= & \sum_{K \in T_{h}} \int_{K} u_{x x}\left(y-y_{K}\right) v_{h y} d x d y+\frac{1}{2} \sum_{K \in T_{h}} \int_{K} u_{x x}\left(y-y_{K}\right)^{2} v_{h y y} d x d y \\
& -\sum_{K \in T_{h}} \frac{h_{y, K}^{2}}{6} \int_{K} u_{x x} v_{h y} d x d y \triangleq B_{1}+B_{2}+B_{3} .
\end{aligned}
$$

Firstly, for all $v_{h y} \in \operatorname{span}\{1, y\}$, we know that

$$
v_{h y}(x, y)=v_{h y}\left(x, y_{K}\right)+\left(y-y_{K}\right) v_{h y} .
$$

Noting that $\left.F(y)\right|_{F_{1}, F_{3}}=0, F^{\prime}(y)=y-y_{K}, F(y)=\frac{1}{6}\left(F^{2}(y)\right)^{\prime \prime}-\frac{h_{y, K}^{2}}{3}$, and by Green's formula, we have

$$
\begin{aligned}
B_{1}= & \sum_{K \in T_{h}} \int_{K} u_{x x} F^{\prime}(y) v_{h y} d x d y \\
= & -\sum_{K \in T_{h}} \int_{K} u_{x x y} F(y) v_{h y} d x d y \\
= & -\sum_{K \in T_{h}} \int_{K} u_{x x y}\left[\frac{1}{6}\left(F^{2}(y)\right)^{\prime \prime}-\frac{h_{y, K}^{2}}{3}\right] v_{h y} d x d y \\
= & \sum_{K \in T_{h}}\left[\frac{1}{6} \int_{K} u_{x x y y}\left(F^{2}(y)\right)^{\prime} v_{h y} d x d y+\int_{K} \frac{h_{y, K}^{2}}{3} v_{h y} d x d y\right] \\
= & \sum_{K \in T_{h}} \frac{1}{6} \int_{K} u_{x x y y}\left(F^{2}(y)\right)^{\prime} v_{h y} d x d y+\sum_{K \in T_{h}} \frac{h_{y, K}^{2}}{3} \int_{K} u_{x x y} v_{h y} d x d y \\
& -\sum_{K \in T_{h}} \frac{h_{y, K}^{2}}{3} \int_{K} u_{x x y}\left(y-y_{K}\right) v_{h y y} d x d y \\
\triangleq & B_{11}+B_{12}+B_{13} .
\end{aligned}
$$

By the inverse inequality, term $B_{11}$ can be estimated as

$$
\begin{aligned}
B_{11} & =\sum_{K \in T_{h}} \frac{1}{6} \int_{K} u_{x x y y}\left(F^{2}(y)\right)^{\prime} v_{h y} d x d y \\
& \leq \sum_{K \in T_{h}} C h_{y, K}^{3}\left\|u_{x x y y}\right\|_{0, K}\left\|v_{h y}\right\|_{0, K} \leq C h^{2}|u|_{4}\left\|v_{h}\right\|_{0} .
\end{aligned}
$$

For the term $B_{12}$, it can be written as

$$
\begin{aligned}
B_{12} & =\sum_{K \in T_{h}} \frac{h_{y, K}^{2}}{3} \int_{K} u_{x x y} v_{h y} d x d y \\
& =\sum_{K \in T_{h}} \frac{h_{y, K}^{2}}{3}\left[\left(\int_{F_{3}}-\int_{F_{1}}\right) u_{x x y} v_{h} d x-\int_{K} u_{x x y y} v_{h} d x d y\right] .
\end{aligned}
$$


Noting that

$$
\begin{aligned}
& \sum_{K \in T_{h}}\left(\int_{F_{3}}-\int_{F_{1}}\right) u_{x x y} v_{h} d x=\sum_{K \in T_{h}}\left(\int_{F_{3}}-\int_{F_{1}}\right) u_{x x y}\left(v_{h}-P_{0} v_{h}\right) d x \\
& =\sum_{K \in T_{h}} \int_{K}\left[u_{x x y y}\left(\left(x-x_{K}\right) v_{h x}+\left(\left(x-x_{K}\right)^{2}+\frac{h_{x, K}^{2}}{3}\right) \frac{v_{h x x}}{2}\right)\right] d x d y \\
& \leq \sum_{K \in T_{h}}\left(C h_{x, K}|u|_{4}\left\|v_{h x}\right\|_{0, K}+C h_{x, K}^{2}|u|_{4}\left\|v_{h x x}\right\|_{0, K}\right) \leq C|u|_{4}\left\|v_{h}\right\|_{0},
\end{aligned}
$$

and substituting (2.10) into (2.9), we can derive

$$
B_{12} \leq C h^{2}|u|_{4}\left\|v_{h}\right\|_{0}
$$

As to the term $B_{13}$, noting that $\left.F(y)\right|_{F_{1}, F_{3}}=0$ and $F^{\prime}(y)=y-y_{K}$, we have

$$
\begin{aligned}
B_{13} & =-\sum_{K \in T_{h}} \frac{h_{y, K}^{2}}{3} \int_{K} u_{x x y} F^{\prime}(y) v_{h y y} d x d y \\
& =\sum_{K \in T_{h}} \frac{h_{y, K}^{2}}{3} \int_{K} u_{x x y y} F(y) v_{h y y} d x d y \leq C h^{2}|u|_{4}\left\|v_{h}\right\|_{0} .
\end{aligned}
$$

Combining with (2.7)-(2.9) and (2.12), we can obtain

$$
B_{1} \leq C h^{2}|u|_{4}\left\|v_{h}\right\|_{0}
$$

Secondly, noting that $\left(y-y_{K}\right)^{2}=\frac{1}{3}\left[\left(F^{2}(y)\right)^{\prime \prime}+h_{y, K}^{2}\right]$, we have

$$
\begin{aligned}
B_{2} & +B_{3} \\
& =\frac{1}{6} \sum_{K \in T_{h}} \int_{K} u_{x x}\left[\left(F^{2}(y)\right)^{\prime \prime}+h_{y, K}^{2}\right] v_{h y y} d x d y-\sum_{K \in T_{h}} \frac{h_{y, K}^{2}}{6} \int_{K} u_{x x} v_{h y y} d x d y \\
& =\frac{1}{6} \sum_{K \in T_{h}} \int_{K} u_{x x}\left(F^{2}(y)\right)^{\prime \prime} v_{h y y} d x d y \frac{1}{6} \sum_{K \in T_{h}} \int_{K} u_{x x y y} F^{2}(y) v_{h y y} d x d y \\
& \leq C h^{4}|u|_{4}\left\|v_{h y y}\right\|_{0} \leq C h^{2}|u|_{4}\left\|v_{h}\right\|_{0} .
\end{aligned}
$$

Finally, substituting estimates (2.13) and (2.14) into (2.6), we obtain

$$
A_{1} \leq C h^{2}|u|_{4}\left\|v_{h}\right\|_{0}
$$

And similarly, we can derive the result

$$
A_{2} \leq C h^{2}|u|_{4}\left\|v_{h}\right\|_{0}
$$

Combining with (2.15) and (2.16), the desired result is obtained. 
Remark 2.1 In [38], the authors derived the following result:

$$
\left|\sum_{K \in T_{h}} \int_{\partial K} \frac{\partial u}{\partial n} v_{h} d s\right| \leq C h^{2}|u|_{5}\left\|v_{h}\right\|_{0}, \quad \forall u \in H^{5}(\Omega), v_{h} \in V_{h} .
$$

Obviously, the regularity requirement of $u$ is stronger than our result.

\section{Backward Euler fully-discrete scheme and superconvergence results}

The variational form of $(1.1)$ is to find $u \in H_{0}^{1}(\Omega)$ such that

$$
\begin{cases}i\left(u_{t}, v\right)-(\nabla u, \nabla v)+\lambda\left(f\left(|u|^{2}\right) u, v\right)=0, & v \in H_{0}^{1}(\Omega), \\ u(X, 0)=u_{0}(X), & X \in \Omega,\end{cases}
$$

where $(u, v)=\int_{\Omega} u \bar{v} d x d y$ denotes the inner product, $\bar{v}$ is the conjugate of $v$.

Given a time step $\tau=T / N$, where $N$ is a positive integer, we shall approximate the solution at times $t_{n}=n \tau, n=0,1, \ldots, N$. For a given smooth function $\phi^{n}$ on $[0, T]$, define $\phi^{n}=\phi\left(X, t^{n}\right), \partial_{t} \phi^{n}=\frac{\phi^{n}-\phi^{n-1}}{\tau}$, and $\partial_{t} \nabla \phi^{n}=\frac{\nabla \phi^{n}-\nabla \phi^{n-1}}{\tau}$.

Equation (3.1) has the following equivalent formulation:

$$
\begin{cases}i\left(\partial_{t} u^{n}, v\right)-\left(\nabla u^{n}, \nabla v\right)+\lambda\left(f\left(\left|u^{n}\right|^{2}\right) u^{n}, v\right)=i\left(R_{1}^{n}, v\right), & v \in H_{0}^{1}(\Omega), \\ u(X, 0)=u_{0}(X), & X \in \Omega,\end{cases}
$$

where $R_{1}^{n}=\partial_{t} u^{n}-u_{t}^{n}$. Furthermore, we have

$$
\begin{aligned}
\left\|R_{1}^{n}\right\|_{0}^{2} & =\left\|\frac{1}{\tau} \int_{t_{n-1}}^{t_{n}}\left(t_{n}-t\right) u_{t t} d t\right\|_{0}^{2} \leq \frac{1}{\tau^{2}} \int_{\Omega}\left(\int_{t_{n-1}}^{t_{n}}\left(t_{n}-t\right) u_{t t} d s\right)^{2} d x d y \\
& \leq \frac{C}{\tau^{2}} \int_{\Omega}\left(\int_{t_{n-1}}^{t_{n}}\left(t_{n}-t\right) d t\right)^{2}\left(\int_{t_{n-1}}^{t_{n}} u_{t t} d s\right)^{2} d x d y \leq C \tau \int_{t_{n-1}}^{t_{n}}\left\|u_{t t}\right\|_{0}^{2} d t .
\end{aligned}
$$

The backward Euler fully-discrete scheme of (3.1) is to find $U^{n} \in V_{h}$ such that

$$
\begin{cases}i\left(\partial_{t} U^{n}, v_{h}\right)-\left(\nabla U^{n}, \nabla v_{h}\right)_{h}+\lambda\left(f\left(\left|U^{n}\right|^{2}\right) U^{n}, v_{h}\right)=0, & v_{h} \in V_{h}, \\ U^{0}=\Pi_{h} u_{0}(X), & X \in \Omega,\end{cases}
$$

where $(\cdot, \cdot)_{h}=\sum_{K \in T_{h}}(\cdot, \cdot)_{K}$.

In order to carry out the error estimate and superclose analysis, we introduce the following assumption.

Assumption 3.1 Let $u^{n}$ and $U^{n}$ be the solutions of (1.1) and (3.4), respectively, for $n=$ $1,2, \ldots, N$, then there exists $0<h_{0}<1$ such that, for $0<h<h_{0}, n=1,2, \ldots, N$, it holds

$$
\left\|u^{n}-U^{n}\right\|_{0, \infty}<1
$$

which means $\left\|U^{n}\right\|_{0, \infty}<C$.

Regarding the proof of Assumption 3.1, one can refer to [15] for details. 
For simplicity, we write

$$
u^{n}-U^{n}=\left(u^{n}-\Pi_{h} u^{n}\right)+\left(\Pi_{h} u^{n}-U^{n}\right) \triangleq \rho^{n}+\theta^{n} .
$$

Then we have the following results.

Theorem 3.1 Assume that $u^{n}$ and $U^{n}$ are the solutions of (1.1) and (3.4), respectively. If $u \in H^{4}(\Omega) \cap H_{0}^{1}(\Omega), u_{t} \in H^{4}(\Omega), u_{t t} \in H^{2}(\Omega), u_{t t t} \in L^{2}(\Omega)$, we have

$$
\left\|\theta^{n}\right\|_{0}+\left\|\theta^{n}\right\|_{h}=O\left(h^{2}+\tau\right)
$$

Proof From (1.1) and (3.4), we have the result

$$
\begin{aligned}
& i\left(\partial_{t}\left(u^{n}-U^{n}\right), v_{h}\right)-\left(\nabla\left(u^{n}-U^{n}\right), v_{h}\right)_{h}+\lambda\left(f\left(\left|u^{n}\right|^{2}\right) u^{n}-f\left(\left|U^{n}\right|^{2}\right) U^{n}, v_{h}\right) \\
& \quad=i\left(R_{1}^{n}, v_{h}\right)-\sum_{K \in T_{h}} \int_{\partial K} \frac{\partial u^{n}}{\partial n} \cdot \overline{v_{h}} d s,
\end{aligned}
$$

which can be rewritten as

$$
\begin{aligned}
i\left(\partial_{t} \theta^{n}, v_{h}\right)-\left(\nabla \theta^{n}, \nabla v_{h}\right)_{h}= & -\lambda\left(f\left(\left|u^{n}\right|^{2}\right) u^{n}-f\left(\left|U^{n}\right|^{2}\right) U^{n}, v_{h}\right) \\
& -i\left(\partial_{t} \rho^{n}, v_{h}\right)+i\left(R_{1}^{n}, v_{h}\right)-\sum_{K \in T_{h}} \int_{\partial K} \frac{\partial u^{n}}{\partial n} \cdot \overline{v_{h}} d s .
\end{aligned}
$$

Taking $v_{h}=\theta^{n}$ in (3.8), we have

$$
\begin{aligned}
i\left(\partial_{t} \theta^{n}, \theta^{n}\right)-\left(\nabla \theta^{n}, \nabla \theta^{n}\right)_{h}= & -\lambda\left(f\left(\left|u^{n}\right|^{2}\right) u^{n}-f\left(\left|U^{n}\right|^{2}\right) U^{n}, \theta^{n}\right) \\
& -i\left(\partial_{t} \rho^{n}, \theta^{n}\right)+i\left(R_{1}^{n}, \theta^{n}\right)-\sum_{K \in T_{h}} \int_{\partial K} \frac{\partial u^{n}}{\partial n} \cdot \overline{\theta^{n}} d s .
\end{aligned}
$$

Comparing the imaginary parts of (3.9), we get

$$
\begin{aligned}
\frac{1}{\tau} \operatorname{Re}\left(\theta^{n}-\theta^{n-1}, \theta^{n}\right) \\
=\frac{1}{2 \tau}\left(\left\|\theta^{n}\right\|_{0}^{2}-\left\|\theta^{n-1}\right\|_{0}^{2}+\left\|\theta^{n}-\theta^{n-1}\right\|_{0}^{2}\right) \\
=-\operatorname{Re}\left(\partial_{t} \rho^{n}, \theta^{n}\right)-\operatorname{Im} \lambda\left(f\left(\left|u^{n}\right|^{2}\right) u^{n}-f\left(\left|U^{n}\right|^{2}\right) U^{n}, \theta^{n}\right) \\
\quad+\operatorname{Re}\left(R_{1}^{n}, \theta^{n}\right)-\operatorname{Im} \sum_{K \in T_{h}} \int_{\partial K} \frac{\partial u^{n}}{\partial n} \cdot \overline{\theta^{n}} d s,
\end{aligned}
$$

which implies

$$
\begin{aligned}
\left\|\theta^{n}\right\|_{0}^{2}-\left\|\theta^{n-1}\right\|_{0}^{2} & \\
\leq & -2 \tau \operatorname{Re}\left(\partial_{t} \rho^{n}, \theta^{n}\right)-2 \tau \operatorname{Im} \lambda\left(f\left(\left|u^{n}\right|^{2}\right) u^{n}-f\left(\left|U^{n}\right|^{2}\right) U^{n}, \theta^{n}\right) \\
& +2 \tau \operatorname{Re}\left(R_{1}^{n}, \theta^{n}\right)-2 \tau \operatorname{Im} \sum_{K \in T_{h}} \int_{\partial K} \frac{\partial u^{n}}{\partial n} \cdot \overline{\theta^{n}} d s \triangleq \sum_{i=1}^{4} D_{i} .
\end{aligned}
$$


Now, we start to estimate each term $D_{i}(i=1,2,3,4)$ one by one.

Applying $\varepsilon$-Young's inequality, we obtain

$$
\left|D_{1}\right| \leq\left|2 \tau\left(\partial_{t} \rho^{n}, \theta^{n}\right)\right| \leq C h^{4} \int_{t_{n-1}}^{t_{n}}\left|u_{t}\right|_{2}^{2} d t+C \tau\left\|\theta^{n}\right\|_{0}^{2}
$$

By using the continuity of $f(s)$ and Assumption 3.1, we have

$$
\begin{aligned}
\left|D_{2}\right| \leq & 2 \tau|\lambda|\left|\left(f\left(\left|u^{n}\right|^{2}\right) u^{n}-f\left(\left|U^{n}\right|^{2}\right) U^{n}, \theta^{n}\right)\right| \\
= & 2 \tau|\lambda|\left|\left(f\left(\left|u^{n}\right|^{2}\right) u^{n}-f\left(\left|u^{n}\right|^{2}\right) U^{n}+f\left(\left|u^{n}\right|^{2}\right) U^{n}-f\left(\left|U^{n}\right|^{2}\right) U^{n}, \theta^{n}\right)\right| \\
= & C \tau\left\|f\left(\left|u^{n}\right|^{2}\right)\right\|_{0, \infty}\left|\left(\rho^{n}+\theta^{n}, \theta^{n}\right)\right| \\
& +C \tau\left\|U^{n}\right\|_{0, \infty}\left\|f^{\prime}\left(\xi_{1}\right)\right\|_{0, \infty}\left(\left\|u^{n}\right\|_{0, \infty}+\left\|U^{n}\right\|_{0, \infty}\right)\left|\left(\rho^{n}+\theta^{n}, \theta^{n}\right)\right| \\
\leq & C \tau\left|\left(\rho^{n}+\theta^{n}, \theta^{n}\right)\right| \leq C \tau h^{4}\left|u^{n}\right|_{2}^{2}+C \tau\left\|\theta^{n}\right\|_{0}^{2},
\end{aligned}
$$

where $\xi_{1}$ lies between $\left|u^{n}\right|^{2}$ and $\left|U^{n}\right|^{2}$.

With the help of the result (3.3) and Lemma 2.2, terms $D_{3}$ and $D_{4}$ can be estimated as

$$
\begin{aligned}
& \left|D_{3}\right| \leq 2 \tau\left|\left(R_{1}^{n}, \theta^{n}\right)\right| \leq 2 \tau\left\|R_{1}^{n}\right\|_{0}\left\|\theta^{n}\right\|_{0} \leq C \tau^{2} \int_{t_{n-1}}^{t_{n}}\left\|u_{t t}\right\|_{0}^{2} d t+C \tau\left\|\theta^{n}\right\|_{0}^{2}, \\
& \left|D_{4}\right| \leq C \tau h^{4}\left|u^{n}\right|_{4}\left\|\theta^{n}\right\|_{0} \leq C \tau h^{4}\left|u^{n}\right|_{4}^{2}+C \tau\left\|\theta^{n}\right\|_{0}^{2} .
\end{aligned}
$$

Combining the above estimates (3.11)-(3.15) yields

$$
\begin{aligned}
\left\|\theta^{n}\right\|_{0}^{2}-\left\|\theta^{n-1}\right\|_{0}^{2} \leq & C h^{4}\left(\tau\left\|u^{n}\right\|_{4}^{2}+\int_{t_{n-1}}^{t_{n}}\left|u_{t}\right|_{2}^{2} d t\right) \\
& +C \tau^{2} \int_{t_{n-1}}^{t_{n}}\left\|u_{t t}\right\|_{0}^{2} d t+C \tau\left\|\theta^{n}\right\|_{0}^{2}
\end{aligned}
$$

Summing (3.16) up with respect to $n$ and noting $\theta^{0}=0$, we have

$$
\begin{aligned}
\left\|\theta^{n}\right\|_{0}^{2} \leq & C h^{4} \int_{0}^{T}\left|u_{t}\right|_{2}^{2} d t+C \tau^{2} \int_{0}^{T}\left\|u_{t t}\right\|_{0}^{2} d t \\
& +C \tau h^{4} \sum_{i=1}^{n}\left\|u^{i}\right\|_{4}^{2}+C \tau \sum_{i=1}^{n}\left\|\theta^{i}\right\|_{0}^{2} .
\end{aligned}
$$

By Gronwall's lemma, we can derive

$$
\left\|\theta^{n}\right\|_{0}^{2} \leq C h^{4} \int_{0}^{T}\left|u_{t}\right|_{2}^{2} d t+C \tau^{2} \int_{0}^{T}\left\|u_{t t}\right\|_{0}^{2} d t+C \tau h^{4} \sum_{i=1}^{n}\left\|u^{i}\right\|_{4}^{2},
$$

which implies

$$
\left\|\theta^{n}\right\|_{0}=O\left(h^{2}+\tau\right) .
$$


On the other hand, taking $v_{h}=\partial_{t} \theta^{n}$ in (3.8), we obtain

$$
\begin{aligned}
& i\left(\partial_{t} \theta^{n}, \partial_{t} \theta^{n}\right)-\left(\nabla \theta^{n}, \nabla \partial_{t} \theta^{n}\right)_{h} \\
& =-\lambda\left(f\left(\left|u^{n}\right|^{2}\right) u^{n}-f\left(\left|U^{n}\right|^{2}\right) U^{n}, \partial_{t} \theta^{n}\right) \\
& \quad-i\left(\partial_{t} \rho^{n}, \partial_{t} \theta^{n}\right)+i\left(R_{1}^{n}, \partial_{t} \theta^{n}\right)-\sum_{K \in T_{h}} \int_{\partial K} \frac{\partial u}{\partial n} \cdot \overline{\partial_{t} \theta^{n}} d s .
\end{aligned}
$$

Comparing the real parts of (3.20), we get

$$
\begin{aligned}
\frac{1}{\tau} & \operatorname{Re}\left(\nabla \theta^{n}, \nabla \theta^{n}-\nabla \theta^{n-1}\right)_{h} \\
= & \frac{1}{2 \tau}\left(\left\|\nabla \theta^{n}\right\|_{0}^{2}-\left\|\nabla \theta^{n-1}\right\|_{0}^{2}+\left\|\nabla \theta^{n}-\nabla \theta^{n-1}\right\|_{0}^{2}\right) \\
= & -\operatorname{Im}\left(\partial_{t} \rho^{n}, \partial_{t} \theta^{n}\right)+\operatorname{Re} \lambda\left(f\left(\left|u^{n}\right|^{2}\right) u^{n}-f\left(\left|U^{n}\right|^{2}\right) U^{n}, \partial_{t} \theta^{n}\right) \\
& +\operatorname{Im}\left(R_{1}^{n}, \partial_{t} \theta^{n}\right)+\operatorname{Re} \sum_{K \in T_{h}} \int_{\partial K} \frac{\partial u^{n}}{\partial n} \cdot \overline{\partial_{t} \theta^{n}} d s,
\end{aligned}
$$

which implies

$$
\begin{aligned}
\left\|\theta^{n}\right\|_{h}^{2}-\left\|\theta^{n-1}\right\|_{h}^{2} & \\
\leq & -2 \tau \operatorname{Im}\left(\partial_{t} \rho^{n}, \partial_{t} \theta^{n}\right)+2 \tau \operatorname{Re} \lambda\left(f\left(\left|u^{n}\right|^{2}\right) u^{n}-f\left(\left|U^{n}\right|^{2}\right) U^{n}, \partial_{t} \theta^{n}\right) \\
& +2 \tau \operatorname{Im}\left(R_{1}^{n}, \partial_{t} \theta^{n}\right)+2 \tau \operatorname{Re} \sum_{K \in T_{h}} \int_{\partial K} \frac{\partial u^{n}}{\partial n} \cdot \overline{\partial_{t} \theta^{n}} d s \\
\leq & C \tau h^{4}\left(\left\|u^{n}\right\|_{4}^{2}+\int_{t_{n-1}}^{t_{n}}\left|u_{t}\right|_{2}^{2} d t\right)+C \tau^{2} \int_{t_{n-1}}^{t_{n}}\left\|u_{t t}\right\|_{0}^{2} d t+C \tau\left\|\partial_{t} \theta^{n}\right\|_{0}^{2} .
\end{aligned}
$$

Now we estimate the term $\left\|\partial_{t} \theta^{n}\right\|_{0}^{2}$. To do this, take difference between two time levels $n$ and $n-1$ of (3.8) and multiply by $\frac{1}{\tau}$ on both sides, then set $v_{h}=\partial_{t} \theta^{n}$ to get

$$
\begin{aligned}
& i\left(\partial_{t}\left(\partial_{t} \theta^{n}\right), \partial_{t} \theta^{n}\right)-\left(\nabla \partial_{t} \theta^{n}, \nabla \partial_{t} \theta^{n}\right) \\
& =-\lambda\left(\partial_{t} M^{n}, \partial_{t} \theta^{n}\right)-i\left(\partial_{t}\left(\partial_{t} \rho^{n}\right), \partial_{t} \theta^{n}\right) \\
& \quad+i\left(\partial_{t} R_{1}^{n}, \partial_{t} \theta^{n}\right)-\sum_{K \in T_{h}} \int_{\partial K} \frac{\partial\left(\partial_{t} u^{n}\right)}{\partial n} \cdot \overline{\partial_{t} \theta^{n}} d s,
\end{aligned}
$$

where $M^{n}=f\left(\left|u^{n}\right|^{2}\right) u^{n}-f\left(\left|U^{n}\right|^{2}\right) U^{n}$.

From [15], we know that

$$
\begin{aligned}
& \left\|\partial_{t} M^{n}\right\|_{0}^{2} \leq C\left(\left\|\partial_{t} \theta^{n}\right\|_{0}^{2}+\left\|\partial_{t} \eta^{n}\right\|_{0}^{2}+\left\|\eta^{n-1}\right\|_{0}^{2}+\left\|\theta^{n-1}\right\|_{0}^{2}\right), \\
& \left\|\partial_{t}\left(\partial_{t} \rho^{n}\right)\right\|_{0}^{2} \leq \frac{C h^{4}}{\tau} \int_{t_{n-2}}^{t_{n}}\left|u_{t t}\right|_{2}^{2} d t, \\
& \left\|\partial_{t} R_{1}^{n}\right\|_{0}^{2} \leq C \tau \int_{t_{n-2}}^{t_{n}}\left|u_{t t t}\right|_{0}^{2} d t .
\end{aligned}
$$


Further, we have by Lemma 2.2 that

$$
\begin{aligned}
\left|\sum_{K \in T_{h}} \int_{\partial K} \frac{\partial\left(\partial_{t} u^{n}\right)}{\partial n} \cdot \overline{\partial_{t} \theta^{n}} d s\right| & \leq C h^{2}\left|\partial_{t} u^{n}\right|_{4}\left\|\partial_{t} \theta^{n}\right\|_{0} \\
& \leq C h^{4}\left(\left|\partial_{t} u^{n}\right|_{4}^{2}+\left\|\partial_{t} \theta^{n}\right\|_{0}^{2}\right) \\
& \leq C h^{4}\left(\frac{1}{\tau} \int_{t_{n-1}}^{t_{n}}\left\|u_{t}\right\|_{4}^{2} d t+\left\|\partial_{t} \theta^{n}\right\|_{0}^{2}\right)
\end{aligned}
$$

Comparing the imaginary part of (3.23) with the above estimations, we obtain

$$
\begin{aligned}
& \frac{\left\|\partial_{t} \theta^{n}\right\|_{0}^{2}-\left\|\partial_{t} \theta^{n-1}\right\|_{0}^{2}}{2 \tau} \\
& \leq \frac{C h^{4}}{\tau} \int_{t_{n-2}}^{t_{n}}\left|u_{t t}\right|_{2}^{2} d t+C \tau \int_{t_{n-2}}^{t_{n}}\left|u_{t t t}\right|_{0}^{2} d t+\frac{C h^{4}}{\tau} \int_{t_{n-1}}^{t_{n}}\left\|u_{t}\right\|_{4}^{2} d t \\
& \quad+\left\|\eta^{n-1}\right\|_{0}^{2}+\left\|\theta^{n-1}\right\|_{0}^{2}+C\left\|\partial_{t} \theta^{n}\right\|_{0}^{2} .
\end{aligned}
$$

Summing (3.28) up with respect to $n$ leads to

$$
\begin{aligned}
\left\|\partial_{t} \theta^{n}\right\|_{0}^{2} \leq & C h^{4} \int_{0}^{T}\left(\left|u_{t t}\right|_{2}^{2}+\left\|u_{t}\right\|_{4}^{2}\right) d t+C \tau^{2} \int_{0}^{T}\left|u_{t t t}\right|_{0}^{2} d t \\
& +C \tau \sum_{i=2}^{n}\left(\left\|\eta^{i-1}\right\|_{0}^{2}+\left\|\theta^{i-1}\right\|_{0}^{2}\right)+C \sum_{i=2}^{n}\left\|\partial_{t} \theta^{i}\right\|_{0}^{2}+\left\|\partial_{t} \theta^{1}\right\|_{0}^{2} .
\end{aligned}
$$

Setting $n=1$ and taking $v_{h}=\partial_{t} \theta^{1}$ in (3.8), with an argument similar to (3.19), we can derive that

$$
\left\|\partial_{t} \theta^{1}\right\|_{0} \leq C\left(h^{2}+\tau\right)
$$

which together with (3.19), (3.29), and (3.30) gives

$$
\left\|\partial_{t} \theta^{n}\right\|_{0} \leq C\left(h^{2}+\tau\right)
$$

Substituting (3.31) into (3.22) and summing up from 1 to $n$ yields

$$
\left\|\theta^{n}\right\|_{h}=O\left(h^{2}+\tau\right)
$$

Thus the proof is complete.

Now we will introduce a proper interpolation postprocessing operator to get the global superconvergence result. For this purpose, we further assume that $T_{h}$ has been obtained from $T_{2 h}$ by dividing each element into four congruent rectangles. Let $\mathcal{T}=\bigcup_{i=1}^{4} K_{i}, L_{1}, L_{2}$, $L_{3}$, and $L_{4}$ be four edges. As in $[31,38]$, we define the interpolation operator $\Pi_{2 h}$ on the partition $T_{2 h}$ :

$$
\left\{\begin{array}{l}
\left.\Pi_{2 h} u\right|_{\mathcal{T}} \in P_{2}(\mathcal{T}), \quad \forall \mathcal{T} \in T_{2 h} \\
\int_{L_{i}}\left(\Pi_{2 h} u-u\right) d s=0, \quad i=1,2,3,4 \\
\int_{K_{1} \cup K_{3}}\left(\Pi_{2 h} u-u\right) d x d y=0, \quad \int_{K_{2} \cup K_{4}}\left(\Pi_{2 h} u-u\right) d x d y=0
\end{array}\right.
$$

where $P_{2}(\mathcal{T})$ denotes the set of polynomials of degree 2 . 
It has been shown in [38] that the interpolation operator $\Pi_{2 h}$ defined above satisfies the following properties:

$$
\begin{aligned}
& \Pi_{2 h} \Pi_{h} u=\Pi_{2 h} u, \quad\left\|u-\Pi_{2 h} u\right\|_{h} \leq C h^{r}|u|_{r+1, \Omega}, \quad 0 \leq r \leq 2, \\
& \left\|\Pi_{2 h} v\right\|_{h} \leq C\|v\|_{h}, \quad \forall v \in V_{h} .
\end{aligned}
$$

Theorem 3.2 Under the same assumptions of Theorem 3.1, we have

$$
\left\|u^{n}-\Pi_{2 h} U^{n}\right\|_{h}=O\left(h^{2}+\tau\right)
$$

Proof Noticing that

$$
u^{n}-\Pi_{2 h} U^{n}=u^{n}-\Pi_{2 h} \Pi_{h} u^{n}+\Pi_{2 h} \Pi_{h} u^{n}-\Pi_{2 h} U^{n},
$$

by (3.33) and interpolation error estimates, we have

$$
\left\|u^{n}-\Pi_{2 h} \Pi_{h} U^{n}\right\|_{h}=\left\|u^{n}-\Pi_{2 h} u^{n}\right\|_{h} \leq C h^{2}\left|u^{n}\right|_{3} .
$$

Consequently, it follows from (3.34) and Theorem 3.1 that

$$
\begin{aligned}
\left\|\Pi_{2 h} \Pi_{h} u^{n}-\Pi_{2 h} U^{n}\right\|_{h} & =\left\|\Pi_{2 h}\left(\Pi_{h} u^{n}-U^{n}\right)\right\|_{h} \\
& \leq\left\|\Pi_{h} u^{n}-U^{n}\right\|_{h}=O\left(h^{2}+\tau\right) .
\end{aligned}
$$

From (3.36)-(3.38), we can derive the result (3.35) directly.

Remark 3.1 Theorems 3.1-3.2 are also valid to the $Q_{1}^{\text {rot }}$ element [39] on square meshes.

\section{The two-grid finite element scheme and error analysis}

In this section, we design a two-grid finite element algorithm (see Algorithm 4.1 below) for problem (1.1) to reduce the computing cost. The idea of the two-grid method is to reduce the nonlinear problem on a fine grid into a linear system through solving a nonlinear problem on a coarse grid. $T_{H}$ and $T_{h}$ are two regular subdivisions of $\Omega$ with two different mesh sizes $H$ and $h(h \ll H)$, and the corresponding $E Q_{1}^{\text {rot }}$ finite element spaces $V_{H}$ and $V_{h}$ (which will be called the coarse-grid space and the fine-grid space), respectively.

\section{Algorithm 4.1}

Step 1. Find $u_{H}^{n} \in V_{H}(n=1,2, \ldots, N)$ such that

$$
\begin{cases}i\left(\partial_{t} u_{H}^{n}, v_{H}\right)-\left(\nabla u_{H}^{n}, \nabla v_{H}\right)+\lambda\left(f\left(\left|u_{H}^{n}\right|^{2}\right) u_{H}^{n}, v_{H}\right)=0, & v_{H} \in V_{H}, \\ u_{H}^{0}=\Pi_{h} u_{0}(X) \in V_{H}, & X \in \Omega .\end{cases}
$$

Step 2. Find $u_{h}^{n} \in V_{h}(n=1,2, \ldots, N)$ such that

$$
\begin{cases}\left.i\left(\partial_{t} u_{h}^{n}, v_{h}\right)-\left(\nabla u_{h}^{n}, \nabla v_{h}\right)+\lambda\left(\widetilde{f\left(\left|u_{H}^{n}\right|^{2}\right.}\right) u_{h}^{n}, v_{h}\right)=0, & v_{h} \in V_{h}, \\ u_{h}^{0}=\Pi_{h} u_{0}(X) \in V_{h}, & X \in \Omega,\end{cases}
$$

where $\left.f \widetilde{\left(\left|u_{H}^{n}\right|^{2}\right.}\right)=f\left(\left|u_{H}^{n}\right|^{2}\right)+f^{\prime}\left(\left|u_{H}^{n}\right|^{2}\right)\left(\left|u_{h}^{n-1}\right|^{2}-\left|u_{H}^{n}\right|^{2}\right)$. 
Now we consider the error estimates in the broken $H^{1}$-norm for Algorithm 4.1.

Theorem 4.1 Let $u$ and $u_{h}^{n}$ be the solutions of problem (1.1) and the two-grid Algorithm 4.1, respectively. If $u \in H^{4}(\Omega) \cap W^{2, \infty}(\Omega) \cap H_{0}^{1}(\Omega), u_{t} \in H^{4}(\Omega), u_{t t} \in H^{2}(\Omega)$, and $u_{t t t} \in L^{2}(\Omega)$, there holds

$$
\left\|\theta^{n}\right\|_{0}+\left\|\theta^{n}\right\|_{h}=O\left[h^{2}+\tau+H^{4}|\ln H|^{\frac{1}{2}}\right]
$$

Proof From (1.1) and (4.2), similar to (3.11), we have the result

$$
\begin{aligned}
\left\|\theta^{n}\right\|_{0}^{2}-\left\|\theta^{n-1}\right\|_{0}^{2} & \\
= & -2 \tau \operatorname{Re}\left(\partial_{t} \rho^{n}, \theta^{n}\right)-\operatorname{Im} \lambda\left(f\left(\left|u^{n}\right|^{2}\right) u_{h}^{n}-f\left(\left|u_{H}^{n}\right|^{2}\right) u_{h}^{n}, \theta^{n}\right) \\
& +2 \tau \operatorname{Re}\left(R_{1}^{n}, \theta^{n}\right)-2 \tau \operatorname{Im} \sum_{K \in T_{h}} \int_{\partial K} \frac{\partial u^{n}}{\partial n} \cdot \overline{\theta^{n}} d s \triangleq \sum_{i=1}^{4} M_{i} .
\end{aligned}
$$

We only need to estimate the term $M_{2}$. In fact, by using the continuity of $f(s)$ and Taylor expansions, we have

$$
f\left(\left|u^{n}\right|^{2}\right)=f\left(\left|u_{H}^{n}\right|^{2}\right)+f^{\prime}\left(\left|u_{H}^{n}\right|^{2}\right)\left(\left|u^{n}\right|^{2}-\left|u_{H}^{n}\right|^{2}\right)+\frac{f^{\prime \prime}\left(\xi_{2}\right)}{2 !}\left(\left|u^{n}\right|^{2}-\left|u_{H}^{n}\right|^{2}\right)^{2},
$$

where $\xi_{1}$ lies between $\left|u^{n}\right|^{2}$ and $\left|u_{H}^{n}\right|^{2}$.

Then $M_{2}$ can be expressed as

$$
\begin{aligned}
\left|M_{2}\right| \leq & \left.2 \tau|\lambda| \mid\left(f\left(\left|u^{n}\right|^{2}\right) u^{n}-\widetilde{f\left(\left|u_{H}^{n}\right|^{2}\right.}\right) u_{h}^{n}, \theta^{n}\right) \mid \\
= & \left.\left.\left.2 \tau|\lambda| \mid\left(f\left(\left|u^{n}\right|^{2}\right) u^{n}-\widetilde{f\left(\left|u_{H}^{n}\right|^{2}\right.}\right) u^{n}+\widetilde{\left(\left|u_{H}^{n}\right|^{2}\right.}\right) u^{n}-\widetilde{f\left(\left|u_{H}^{n}\right|^{2}\right.}\right) u_{h}^{n}, \theta^{n}\right) \mid \\
= & \left.2 \tau|\lambda| \mid\left(f\left(\left|u^{n}\right|^{2}\right) u^{n}-\widetilde{\left(\left|u_{H}^{n}\right|^{2}\right.}\right) u^{n}, \theta^{n}\right) \mid \\
& +2 \tau|\lambda|\left|\left(\widetilde{\left(\left|u_{H}^{n}\right|^{2}\right)}\left(u^{n}-u_{h}^{n}\right), \theta^{n}\right)\right| \triangleq E_{1}+E_{2} .
\end{aligned}
$$

Firstly, for the term $E_{1}$, we have

$$
\begin{aligned}
E_{1}= & 2 \tau|\lambda|\left|\left(\left[f^{\prime}\left(\left|u_{H}^{n}\right|^{2}\right)\left(\left|u^{n}\right|^{2}-\left|u_{h}^{n-1}\right|^{2}\right)+\frac{f^{\prime \prime}\left(\left|\xi_{2}\right|^{2}\right)}{2}\left(\left|u^{n}\right|^{2}-\left|u_{H}^{n}\right|^{2}\right)^{2}\right] u^{n}, \theta^{n}\right)\right| \\
= & 2 \tau|\lambda|\left|\left(f^{\prime}\left(\left|u_{H}^{n}\right|^{2}\right)\left(\left|u^{n}\right|^{2}-\left|u^{n-1}\right|^{2}\right) u^{n}, \theta^{n}\right)\right| \\
& +2 \tau|\lambda|\left|\left(f^{\prime}\left(\left|u_{H}^{n}\right|^{2}\right)\left(\left|u^{n-1}\right|^{2}-\left|u_{h}^{n-1}\right|^{2}\right) u^{n}, \theta^{n}\right)\right| \\
& +\tau|\lambda|\left|\left(f^{\prime \prime}\left(\left|\xi_{2}\right|^{2}\right)\left(\left|u^{n}\right|^{2}-\left|u_{H}^{n}\right|^{2}\right)^{2} u^{n}, \theta^{n}\right)\right| \\
\triangleq & E_{11}+E_{12}+E_{13} .
\end{aligned}
$$

Applying the boundedness of $u, f(s)$, and Theorem 3.1, we can derive that

$$
E_{11}+E_{12} \leq C \tau\left(h^{4}+\tau^{2}\right)
$$


Similar to (3.13), $E_{13}$ can be estimated as

$$
E_{13} \leq C \tau\left(\left\|\left(\left|u^{n}\right|^{2}-\left|u_{H}^{n}\right|^{2}\right)^{2}\right\|_{0}^{2}+\left\|\theta^{n}\right\|_{0}^{2}\right)
$$

Notice that the term $\left\|\left(\left|u^{n}\right|^{2}-\left|u_{H}^{n}\right|^{2}\right)^{2}\right\|_{0}^{2}$ can be rewritten as

$$
\begin{aligned}
\left\|\left(\left|u^{n}\right|^{2}-\left|u_{H}^{n}\right|^{2}\right)^{2}\right\|_{0}^{2} & \leq C\left\|\left(\left|u^{n}\right|^{2}-\left|u_{H}^{n}\right|^{2}\right)^{2}\right\|_{0}^{2} \leq C\left\|u^{n}-u_{H}^{n}\right\|_{0, \infty}^{2}\left\|u^{n}-u_{H}^{n}\right\|_{0}^{2} \\
& \leq\left(\left\|u^{n}-\Pi_{H} u^{n}\right\|_{0, \infty}^{2}+\left\|\Pi_{H} u^{n}-u_{H}^{n}\right\|_{0, \infty}^{2}\right)\left\|u^{n}-u_{H}^{n}\right\|_{0}^{2} .
\end{aligned}
$$

Since

$$
\begin{aligned}
& \left\|u^{n}-\Pi_{H} u^{n}\right\|_{0, \infty}^{2} \leq C H^{4}\left\|u^{n}\right\|_{2, \infty}^{2}, \\
& \left\|u^{n}-u_{H}^{n}\right\|_{0}^{2} \leq C\left(H^{4}+\tau^{2}\right),
\end{aligned}
$$

and $\left\|v_{h}\right\|_{0, \infty} \leq C|\ln h|^{\frac{1}{2}}\left\|v_{h}\right\|_{h}[40]$, we have

$$
\left\|\Pi_{H} u^{n}-u_{H}^{n}\right\|_{0, \infty}^{2} \leq C|\ln H|\left\|\Pi_{H} u^{n}-u_{H}^{n}\right\|_{h}^{2} \leq C|\ln H|\left(H^{4}+\tau^{2}\right) .
$$

Then from (4.10)-(4.13), we know that

$$
\left\|\left(\left|u^{n}\right|^{2}-\left|u_{H}^{n}\right|^{2}\right)^{2}\right\|_{0}^{2} \leq C\left[H^{4}+\tau^{2}+|\ln H|\left(H^{4}+\tau^{2}\right)\right]\left(H^{4}+\tau^{2}\right) .
$$

Further, when $\tau$ is small enough, there holds

$$
\left\|\left(\left|u^{n}\right|^{2}-\left|u_{H}^{n}\right|^{2}\right)^{2}\right\|_{0}^{2} \leq C H^{8}|\ln H|
$$

which implies

$$
E_{1} \leq C \tau\left(h^{4}+\tau^{2}+H^{8}|\ln H|\right)
$$

Secondly, for the term $E_{2}$, we have

$$
E_{2} \leq C \tau\left(\left\|u^{n}-u_{h}^{n}\right\|_{0}^{2}+\left\|\theta^{n}\right\|_{0}^{2}\right) \leq C \tau\left(h^{4}+\tau^{2}\right) .
$$

Finally, substituting (4.16) and (4.17) into (4.6), we obtain

$$
\left|M_{2}\right| \leq C \tau\left(h^{4}+\tau^{2}+H^{8}|\ln H|\right),
$$

and substituting (3.12), (3.14), (3.15), and (4.18) into (4.4) yields

$$
\left\|\theta^{n}\right\|_{0}^{2}-\left\|\theta^{n-1}\right\|_{0}^{2} \leq C \tau\left(h^{4}+\tau^{2}+H^{8}|\ln H|\right)+C \tau\left\|\theta^{n}\right\|_{0}^{2} .
$$

Then summing (4.19) up with respect to $n$ and noting $\theta^{0}=0$, we have

$$
\left\|\theta^{n}\right\|_{0}^{2} \leq C\left(h^{4}+\tau^{2}+H^{8}|\ln H|\right)+C \tau \sum_{i=1}^{n}\left\|\theta^{i}\right\|_{0}^{2} .
$$


An application of Gronwall's lemma yields

$$
\left\|\theta^{n}\right\|_{0}^{2} \leq C\left(h^{4}+\tau^{2}+H^{8}|\ln H|\right),
$$

which implies that

$$
\left\|\theta^{n}\right\|_{0}=O\left(h^{2}+\tau+H^{4}|\ln H|^{\frac{1}{2}}\right) .
$$

Thus with the similar arguments to the estimates of (3.32) and (4.22), we can also derive

$$
\left\|\theta^{n}\right\|_{h}=O\left(h^{2}+\tau+H^{4}|\ln H|^{\frac{1}{2}}\right),
$$

which is the desired result.

Similar to the proof of Theorem 3.2, we can derive the following superconvergence results.

Theorem 4.2 Under the same assumptions of Theorem 4.1, and setting $h=H^{2}(|\ln H|)^{\frac{1}{4}}$, we can derive

$$
\left\|u^{n}-\Pi_{2 h} u_{h}^{n}\right\|_{h}=O\left(h^{2}+\tau\right) .
$$

\section{Numerical experiment}

In this section, we present the following numerical example to confirm the theoretical analysis, which comes from $[8,16,41]$.

Consider the cubic-quintic Schrödinger equation $\left(f(s)=-s+s^{2}\right)$

$$
\begin{cases}i u_{t}+\Delta u-|u|^{2} u+|u|^{4} u=g, & (X, t) \in \Omega \times(0, T] \\ u(X, t)=0, & (X, t) \in \partial \Omega \times(0, T] \\ u(X, 0)=u_{0}(X), & X \in \Omega,\end{cases}
$$

on $\Omega=[0,1]^{2}$ with the exact solution

$$
u=e^{i t+(x+y) / 2}\left(1+3 t^{2}\right) x(1-x) y(1-y)
$$

where $g$ is given corresponding to the exact solution $u$.

The domain $\Omega$ is divided into families $T_{H}$ and $T_{h}$ of rectangular meshes, and $V_{H}, V_{h}$ are $E Q_{1}^{\text {rot }}$ finite element spaces defined on $T_{H}, T_{h}$, respectively. In such a way, to obtain enough accuracy, it suffices to take $h=O\left(H^{2}|\ln H|^{\frac{1}{4}}\right)$ in both the broken $H^{1}$-norm and $L^{2}$-norm. Under the same condition of computing environment and control strategy, the numerical results and CPU times are shown in Tables $1-2$, respectively. The exact solution $u$ at time $t=1$ and FEM solution $u_{h}$ at time $t=1$ with mesh size $h=1 / 32$ are pictured in Figs. 1 and 2 , respectively.

It can be seen from Tables $1-2$ that $\left\|u-u_{h}\right\|_{h}$ is convergence at rate of $O(h),\left\|u-u_{h}\right\|_{0}$, $\left\|\Pi_{h} u-u_{h}\right\|_{h}$ and $\left\|u-\Pi_{2 h} u\right\|_{h}$ are convergence at rate of $O\left(h^{2}\right)$, which coincide with the theoretical analysis. Thus the two-grid FEM is more efficient in solving NLSE than the usual Galerkin FEM. 
Table 1 Numerical results of two-grid Algorithm 4.1 at $t=1\left(\tau=h^{2}, h \approx H^{2}\right)$

\begin{tabular}{lllll}
\hline & $h=\frac{1}{16}, H=\frac{1}{4}$ & $h=\frac{1}{32}, H=\frac{1}{6}$ & $h=\frac{1}{64}, H=\frac{1}{8}$ & Ratio \\
\hline$\left\|u-u_{h}\right\|_{h}$ & 0.058942538 & 0.029468965 & 0.014736033 & 0.99 \\
$\left\|u-u_{h}\right\|_{0}$ & 0.001096657 & 0.000281836 & 0.000075823 & 1.93 \\
$\left\|\Pi_{h} u-u_{h}\right\|_{h}$ & 0.003761590 & 0.000980134 & 0.000264975 & 1.91 \\
$\left\|u-\Pi_{2 h} u_{h}\right\|_{h}$ & 0.018173033 & 0.004582958 & 0.001179143 & 1.97 \\
CPU time $(s)$ & 54.06 & 2973.62 & $237,992.52$ & \\
\hline
\end{tabular}

Table 2 Numerical results of the usual Galerkin FEM at $t=1\left(\tau=h^{2}\right)$

\begin{tabular}{lllll}
\hline & $h=\frac{1}{16}$ & $h=\frac{1}{32}$ & $h=\frac{1}{64}$ & Ratio \\
\hline$\left\|u-u_{h}\right\|_{h}$ & 0.058940220 & 0.029468644 & 0.014523013 & 1.01 \\
$\left\|u-u_{h}\right\|_{0}$ & 0.001090311 & 0.000280127 & 0.000072122 & 1.96 \\
$\left\|\Pi_{h} u-u_{h}\right\|_{h}$ & 0.003725084 & 0.000970437 & 0.000253940 & 1.94 \\
$\left\|u-\Pi_{2 h} u_{h}\right\|_{h}$ & 0.018165504 & 0.004580893 & 0.001067421 & 2.04 \\
$\mathrm{CPU}$ time (s) & 68.88 & 5178.21 & $416,552.35$ & \\
\hline
\end{tabular}
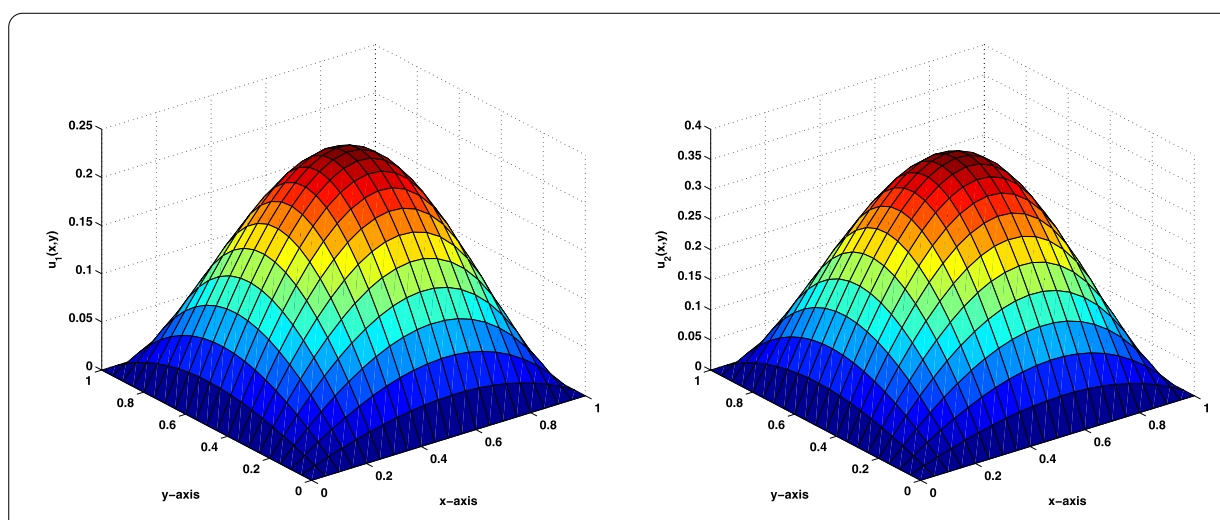

Figure 1 The exact solutions $u_{1}$ (Real part) and $u_{2}$ (Imaginary part) at $t=1$

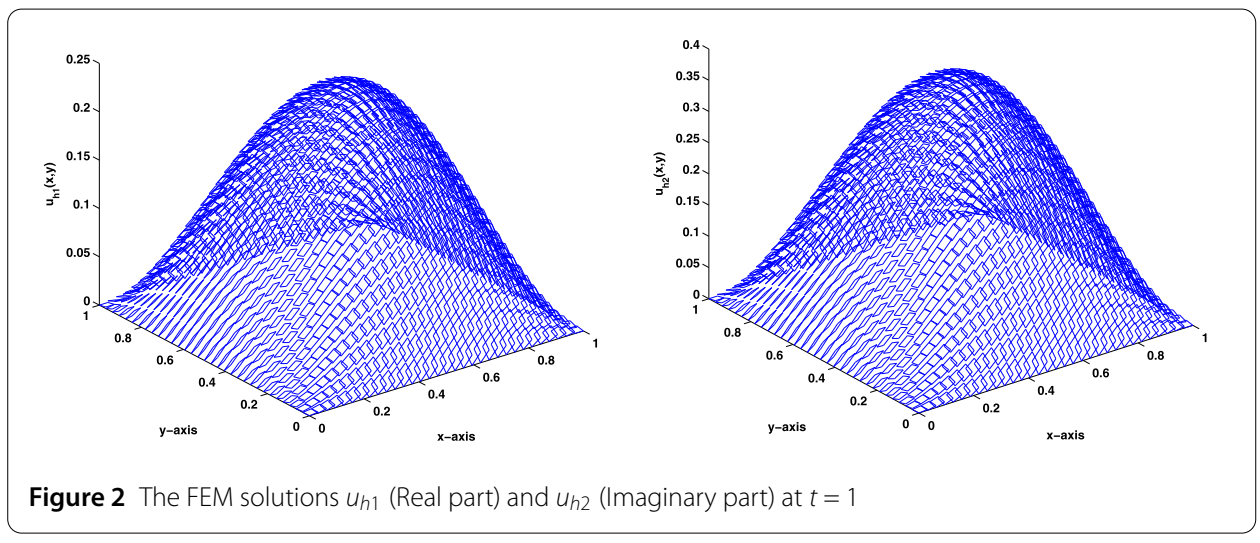

\section{Conclusions}

In this paper, we applied low order nonconforming $E Q_{1}^{\text {rot }}$ finite element to solve the nonlinear Schrödinger equation, and derived the global superconvergence results for the backward Euler fully-discrete scheme and a type of two-grid scheme, respectively. A numerical example is presented to demonstrate the theoretical results. The method presented in this 
paper is suitable for the standard Galerkin finite element and can be extended to dealing with other nonlinear problems.

\author{
Acknowledgements \\ We are thankful to the editor and the anonymous reviewers for many valuable suggestions to improve this paper. \\ Funding \\ The research is supported by the National Natural Science Foundation of China (Nos. 11271340, 11671369), the \\ Educational Commission of Henan Province of China (No. 14B110025).
}

\title{
Abbreviations \\ Not applicable.
}

Availability of data and materials

Not applicable.

\section{Competing interests}

The authors declare that they have no competing interests.

\section{Authors' contributions}

The study was carried out in collaboration among all authors. CX and JQZ carried out the main theorem and wrote the paper together; DYS revised and checked the paper; HCZ checked the article. All authors read and approved the final manuscript.

\section{Author details}

${ }^{1}$ Faculty of Mathematics and Physics Education, Luoyang Institute of Science and Technology, Luoyang, China. ${ }^{2}$ School of Mathematics and Statistics, Zhengzhou University, Zhengzhou, China. ${ }^{3}$ School of Mathematics and Statistics,

Pingdingshan University, Pingdingshan, China.

\section{Publisher's Note}

Springer Nature remains neutral with regard to jurisdictional claims in published maps and institutional affiliations.

Received: 7 April 2018 Accepted: 5 November 2018 Published online: 16 November 2018

\section{References}

1. Reichel, B., Leble, S.: On convergence and stability of a numerical scheme of coupled nonlinear Schrödinger equations. Comput. Math. Appl. 55, 745-759 (2008)

2. Bratsos, A.G.: A modified numerical scheme for the cubic Schrödinger equation. Numer. Methods Partial Differ. Equ. $27,608-620(2011)$

3. Liao, H., Sun, Z., Shi, H.: Error estimate of fourth-order compact scheme for linear Schrödinger equations. SIAM J. Numer. Anal. 47, 4381-4401 (2010)

4. Akrivis, G.D., Dougalis, V.A., Karakashian, O.A.: On fully discrete Galerkin methods of second-order temporal accuracy for the nonlinear Schrödinger equation. Numer. Math. 59, 31-53 (1991)

5. Tourigny, Y.: Optimal $H^{1}$ estimates for two time-discrete Galerkin approximations of a nonlinear Schrödinger equation. IMA J. Numer. Anal. 11, 509-523 (1991)

6. Zouraris, G.E.: On the convergence of a linear two-step finite element method for the nonlinear Schrödinger equation. Modél. Math. Anal. Numér. 35, 389-405 (2001)

7. Liu, Y., Li, H., Wang, J.F.: Error estimates of $H^{1}$-Galerkin mixed finite element method for Schrödinger equation. Appl. Math. J. Chin. Univ. 24, 83-89 (2009)

8. Wang, J.L.: A new error analysis of Crank-Nicolson Galerkin FEMs for a generalized nonlinear Schrödinger equation. J. Sci. Comput. 60, 390-407 (2014)

9. Borzi, A., Decker, E.: Analysis of a leap-frog pseudospectral scheme for the Schrödinger equation. J. Comput. Appl. Math. 193, 65-88 (2006)

10. Dehghan, M., Taleei, A.: Numerical solution of nonlinear Schrödinger equation by using time-space pseudo-spectral method. Numer. Methods Partial Differ. Equ. 26, 979-990 (2010)

11. Antoine, X., Besse, C., Klein, P.: Absorbing boundary conditions for general nonlinear Schrödinger equations. SIAM J. Sci. Comput. 33, 1008-1033 (2011)

12. Lin, Q., Liu, X.Q.: Global superconvergence estimates of finite element method for Schrödinger equation. J. Comput. Math. 16, 521-526 (1998)

13. Shi, D.Y., Wang, P.L., Zhao, Y.M.: Superconvergence analysis of anisotropic linear triangular finite element for nonlinear Schrödinger equation. Appl. Math. Lett. 38, 129-134 (2014)

14. Zhao, Y.M., Shi, D.Y., Wang, P.L.: High accuracy analysis of a new mixed finite element method for nonlinear Schrodinger equation. Math. Numer. Sin. 37, 162-178 (2015)

15. Shi, D.Y., Liao, X., Wang, L.L.: A nonconforming quadrilateral finite element approximation to nonlinear Schrödinger equation. Acta Math. Sci. 37, 584-592 (2017)

16. Wang, J.Y., Huang, Y.Q., Tian, Z.K., Zhou, J.: Superconvergence analysis of finite element method for the time-dependent Schrödinger equation. Comput. Math. Appl. 71, 1960-1972 (2016)

17. Shi, D.Y., Wang, J.J.: Unconditional superconvergence analysis of a Crank-Nicolson Galerkin FEM for nonlinear Schrödinger equation. J. Sci. Comput. 72, 1093-1118 (2017) 
18. Xu, J.C.: A new class of iterative methods for nonselfadjoint or indefinite problems. SIAM J. Numer. Anal. 29, 303-319 (1992)

19. Xu, J.C.: Two-grid discretization techniques for linear and nonlinear PDE. SIAM J. Numer. Anal. 33, 1759-1777 (1996)

20. Marion, M., Xu, J.C.: Error estimates on a new nonlinear Galerkin method based on two-grid finite elements. SIAM J. Numer. Anal. 32, 1170-1184 (1995)

21. Layton, W., Lenferink, W.: Two-level Picard and modified Picard methods for the Navier-Stokes equations. Appl. Math. Comput. 69, 263-274 (1995)

22. Dawson, C.N., Wheeler, M.F., Woodward, C.S.: A two-grid finite difference scheme for nonlinear parabolic equations. SIAM J. Numer. Anal. 35, 435-452 (1998)

23. Xu, J.C., Zhou, A.H.: Local and parallel finite element algorithms based on two-grid discretizations. Math. Comput. 69, $881-909$ (2000)

24. Jin, J., Shu, S., Xu, J.C.: A two-grid discretization method for decoupling systems of partial differential equations. Math. Comput. 75, 1617-1626 (2006)

25. Chen, L., Chen, Y.P.: Two-grid method for nonlinear reaction-diffusion equations by mixed finite element methods. J. Sci. Comput. 49, 383-401 (2011)

26. Chien, C.S., Huang, H.T., Jeng, B.W., Lid, Z.C.: Two-grid discretization schemes for nonlinear Schrödinger equations. J. Comput. Appl. Math. 214, 549-571 (2008)

27. Wu, L., Allen, M.B.: A two-grid method for mixed finite-element solutions of reaction-diffusion equations. Numer. Methods Partial Differ. Equ. 15, 589-604 (1999)

28. Wu, L.: Two-grid strategy for unsteady state nonlinear Schrödinger equations. Int. J. Pure Appl. Math. 68, 465-475 (2011)

29. Wu, L.: Two-grid mixed finite-element methods for nonlinear Schrödinger equations. Numer. Methods Partial Differ. Equ. 28, 63-73 (2012)

30. Jin, J.C., Wei, N., Zhang, H.M.: A two-grid finite-element method for the nonlinear Schrödinger equation. J. Comput. Math. 33, 146-157 (2015)

31. Lin, Q., Tobiska, L., Zhou, A.H.: Superconvergence and extrapolation of non-conforming low order finite elements applied to the Poisson equation. IMA J. Numer. Anal. 25, 160-181 (2005)

32. Shi, D.Y., Mao, S.P., Chen, S.C.: An anisotropic nonconforming finite element with some superconvergence results. J. Comput. Math. 23, 261-274 (2005)

33. Shi, D.Y., Xu, C., Chen, J.H.: Anisotropic nonconforming $E Q_{1}^{\text {rot }}$ quadrilateral finite element approximation to second order elliptic problems. J. Sci. Comput. 56, 637-653 (2013)

34. Shi, D.Y., Xu, C.: $E Q_{1}^{\text {rot }}$ nonconforming finite element approximation to Signorini problem. Sci. China Math. 56 $1301-1311(2013)$

35. Shi, D.Y., Wang, J.J., Yan, F.N.: Unconditional superconvergence analysis for nonlinear parabolic equation with $E Q_{1}^{\text {rot }}$ nonconforming finite element. J. Sci. Comput. 70, 85-111 (2017)

36. Shi, D.Y., Wang, J.J.: Unconditional superconvergence analysis for nonlinear hyperbolic equation with nonconforming finite element. Appl. Math. Comput. 305, 1-16 (2017)

37. Shi, D.Y., Wang, J.J., Yan, F.N.: Superconvergence analysis for nonlinear parabolic equation with $E Q_{1}^{\text {rot }}$ nonconforming finite element. Comput. Appl. Math. 37, 307-327 (2018)

38. Lin, Q., Lin, J.F.: Finite Element Methods: Accuracy and Improvement. Science Press, Beijing (2006)

39. Rannacher, R., Turek, S.: Simple nonconforming quadrilateral Stokes element. Numer. Methods Partial Differ. Equ. 8, 97-111 (1992)

40. Wang, M.: On the inequalities for the maximum norm of nonconforming finite element spaces. Math. Numer. Sin. 12 , 104-107 (1990)

41. Pathria, D.: Exact solutions for a generalized nonlinear Schrödinger equation. Phys. Scr. 39, 673-679 (1989)

\section{Submit your manuscript to a SpringerOpen ${ }^{\circ}$ journal and benefit from:}

- Convenient online submission

- Rigorous peer review

- Open access: articles freely available online

- High visibility within the field

- Retaining the copyright to your article

Submit your next manuscript at $\boldsymbol{s p r i n g e r o p e n . c o m ~}$ 